\title{
Pathotypes of Colletotrichum capsici, the Causal Agent of Chili Anthracnose, in Thailand
}

P. Montri, Center for Agricultural Biotechnology, Kasetsart University, Kamphaeng Saen Campus, Nakhon Pathom 73140 Thailand; P. W. J. Taylor, BioMarka/Center for Plant Health, Faculty of Land and Food Resources, The University of Melbourne, Victoria 3010 Australia; and O. Mongkolporn, Department of Horticulture and Center for Agricultural Biotechnology, Kasetsart University, Kamphaeng Saen Campus, Thailand

\begin{abstract}
Montri, P., Taylor, P. W. J., and Mongkolporn, O. 2009. Pathotypes of Colletotrichum capsici, the causal agent of chili anthracnose, in Thailand. Plant Dis. 93:17-20.

Eleven isolates of Colletotrichum capsici were screened on nine chili genotypes derived from four cultivated species of Capsicum: Capsicum annuum, C. baccatum, C. chinense, and C. frutescens. Host reactions were assessed 9 days after inoculation by microinjection of spores into the pericarp of red fruit. A set of disease scales, with 0 to 9 scores, were developed for anthracnose infection of each Capsicum sp. based on percent lesion size in relation to fruit size, appearance of necrotic or water-soaked tissue, and presence of acervuli. Three pathotypes, PCc1, PCc2, and PCc3, were identified according to differential qualitative infection of fruit of $C$. chinense genotypes $\mathrm{PBC} 932$ and $\mathrm{C} 04714$. PCc1 was the most virulent pathotype, infecting all genotypes of $C$. annuum, $C$. chinense, and $C$. frutescens, whereas PCc3 was the least virulent pathotype, infecting only the genotypes $C$. annuum and $C$. frutescens. Quantitative infection occurred in all chili genotypes except for genotypes of $C$. baccatum, where no infection occurred, demonstrating various levels of aggressiveness of isolates within pathotypes.
\end{abstract}

Chili (Capsicum spp.) is the fourth most important vegetable in the world and the first in Asia, with world production in 2006 at approximately 25.9 million $t$ for fresh chili and 2.8 million t for dry chili (1). Thailand produces $420,000 \mathrm{t}$ of chili fruit from 72,000 ha of chili plants with an approximate value of US\$136 million (4). Anthracnose, caused by Colletotrichum spp., is a serious problem for chili production in the tropics and subtropics worldwide. In Thailand, severe epidemics resulted in yield losses of up to $80 \%$ (7). At least three species of Colletotrichum, Colletotrichum capsici, C. gloeosporioides, and $C$. acutatum, have been identified as important pathogens causing chili anthracnose in Asia. In Thailand, C. capsici and C. acutatum have been reported to be the most important pathogens causing anthracnose of chili, although $C$. acutatum was mostly restricted to the north and north east of Thailand (10).

Management of chili anthracnose mainly has been through the use of fungicides. However, fungicide applications are only partially effective under environmental conditions that are favorable for pathogen infection. Furthermore, fungi-

Corresponding author: P. W. J. Taylor

E-mail: paulwjt@unimelb.edu.au

Accepted for publication 4 September 2008.

doi:10.1094/PDIS-93-1-0017

(c) 2009 The American Phytopathological Society cides are not sustainable, especially in small-holder farming systems in Thailand, due to the high cost and risks to the environment. Breeding for resistance has mainly focused on introgression of resistance from Capsicum chinense and $C$. baccatum into elite susceptible genotypes of $C$. апnиит (5). For breeding programs to successfully develop resistant genotypes, knowledge of the variability and pathotype structure within the pathogen population is important.

Sharma et al. (8) reported the existence of 15 pathotypes of Colletotrichum capsici from the Himachal Pradesh area of northern India based on quantitative differences in lesion development on inoculated fruit of Capsicum annuum genotypes. Than et al. (10) showed pathotype differences within Colletotrichum acutatum isolates from infected strawberry and chili fruit. Isolates from chili were able to infect inoculated fruit of the resistant Capsicum

chinense genotype PBC932, whereas isolates from strawberry were unable to infect this genotype. Both isolates were able to infect the susceptible $C$. annuum chili genotype Bangchang. Pathotype differences in chili were identified based on the size of lesions that developed on inoculated fruit (10).

Fruit size and shape of genotypes from different Capsicum spp. vary considerably; therefore, to standardize host reactions between Capsicum spp., Kanchana-udomkan et al. (2) and Pakdeevaraporn et al. (5) measured host reactions based on lesion size as a proportion of fruit size. The development of disease scales for measuring host reactions that incorporated fruit size and severity of infection (lesion size, presence of acervuli, and necrosis or watersoaked tissue) would be appropriate for assisting in screening many isolates across a range of genotypes from different Capsicum spp.

The aims of this study were to develop a set of disease scales for efficient, accurate measurement of the severity of infection in a range of Capsicum spp. and to identify pathotypes of Colletotrichum capsici based on qualitative differences of infection in differential genotypes of Capsicum spp.

\section{MATERIALS AND METHODS}

C. capsici collection. Ten C. capsici (Syd.) E.J. Butler \& Bisby isolates were collected from chili fruit infected with anthracnose in western Thailand (Kanchanaburi, Nakhon Pathom, Suphan Buri) and one from northern Thailand (Chiang Mai) (Table 1). Isolations and establishment of single-spore cultures were carried out according to the procedures of Photita et al. (6) and Than et al. (10).

Table 1. Eleven single-spore isolates of Colletotrichum capsici used in the chili pathotype study

\begin{tabular}{lll}
\hline No. & Colletotrichum isolate & \multicolumn{1}{c}{ Origin } \\
\hline 1 & F4-2C & Kanchanaburi, western Thailand \\
2 & F4-5A & Kanchanaburi, western Thailand \\
3 & F7-3A & Nakhon Pathom, western Thailand \\
4 & F8-1A & Suphan Buri, western Thailand \\
5 & F8-2A & Suphan Buri, western Thailand \\
6 & F8-3A & Suphan Buri, western Thailand \\
7 & F8-3B & Suphan Buri, western Thailand \\
8 & F8-4C & Suphan Buri, western Thailand \\
9 & F8-5A & Suphan Buri, western Thailand \\
10 & F8-5B & Suphan Buri, western Thailand \\
11 & SKP16 & Chiangmai, northern Thailand \\
\hline
\end{tabular}


Colletotrichum cultures and inoculum preparation. A conidial suspension of each $C$. capsici isolate was prepared from conidia that were harvested from 7-day-old cultures grown on potato dextrose agar (PDA; Difco, Becton, Dickinson and Company, Sparks, MD) under $12 \mathrm{~h}$ of darkness and $12 \mathrm{~h}$ of light at 28 to $30^{\circ} \mathrm{C}$. The $C$. capsici culture plates were flooded with sterilized water and the conidia gently scraped from the culture plate using a sterilized loop. The conidial suspension was adjusted to a concentration of approximately $10^{6}$ conidia/ml.

Chili genotypes. Nine chili genotypes of Capsicum annuum L., C. baccatum L., $C$. chinense Jacq., and C. frutescens $\mathrm{L}$. were screened for reaction to the Colletotrichum capsici isolates. Of the nine genotypes, Bangchang, Jinda, and Kheenoo Suan were Thai commercial cultivars and the rest originated from AVRDC (The World Vegetable Center, Tainan, Taiwan).

Pathogenicity study. Five fruit at redripe stage (40 to 50 days after flowering, depending on genotype) harvested from each chili genotype were inoculated with each $C$. capsici isolate using an injection method. After the calyxes were removed, the fruit were surface sterilized with $1 \%$ (wt/vol) sodium hypochlorite for $5 \mathrm{~min}$ and then rinsed twice with distilled water. Approximately 1,000 spores in $1 \mu \mathrm{l}$ of suspension were injected once into the chili fruit pericarp in the middle of each fruit using a microinjector consisting of a Micro Syringe model 1705 TLL and a dispenser PB600-1 (Hamilton, Switzer- land) to ensure equal inoculum volume in each injection. The needle of the microinjector was $1 \mathrm{~mm}$ in diameter and was fixed at $1 \mathrm{~mm}$ long, ensuring uniform wound size on all fruit. The inoculated fruit were placed in plastic boxes ( 20 by 30 by $10 \mathrm{~cm}$ ) on a tray with $500 \mathrm{ml}$ of water in the bottom and incubated at $28^{\circ} \mathrm{C}, 12 \mathrm{~h}$ of darkness and $12 \mathrm{~h}$ of light, and $100 \%$ relative humidity. After 3 days, lids were removed from the boxes to maintain 70 to $80 \%$ relative humidity.

Anthracnose symptoms at the inoculation site were evaluated at 9 days after inoculation on the basis of the percent lesion size relative to the overall size of the fruit. The size of the lesions was visually estimated following the method of Pakdeevaraporn et al. (5). Data for all isolates and genotypes, except for Capsicum baccatum genotypes, which did not become infected, were included in analysis of variance using factorial (11 isolate by 6 genotype) in a completely randomized design (SPSS software version 13.0, SPSS Inc.; 3) to test for main treatments and interaction effects. Duncan's multiple range and least significant difference tests were used for means separations $(P=0.05)$. Each result was arcsin square root transformed before analysis and the means were back transformed for presentation. All experiments were repeated and gave identical results.

Disease scales were developed for each Capsicum sp., in which disease severity was scored on a 0 -to- 9 scale, where 0 was no infection and 9 was infection greater than $25 \%$.
Pathotypes were identified where qualitative differences in infection (0 versus 1to- 9 scores) occurred for isolates on a specific chili genotype. Differences in lesion size were an indication of the levels of aggressiveness of each isolate.

\section{RESULTS}

Differential reactions of chili genotypes to anthracnose infection. The genotypes of Capsicum spp. used in the bioassay exhibited a range of host reactions to infection by the 11 isolates of Colletotrichum capsici (Table 2). Capsicum frutescens and $C$. annuum were highly susceptible, with mean percent lesion size ranging from $27 \%$ for C. annuum Bangchang to $98 \%$ for $C$. frutescens Kheenoo Suan, with the latter being significantly more susceptible to infection than the three $C$. annuum genotypes. Among the $C$. annuum genotypes, there was a range of disease severity, with 83-168 (89\%) being significantly more susceptible to all isolates than Bangchang (35\%) and Jinda (27\%). The C. chinense genotypes C04714 and PBC932 were susceptible (19\%) to moderately susceptible $(12 \%)$, respectively. However, within each of the two $C$. chinense genotypes, there was a range of disease severity reactions from 0 to $59 \%$ lesion size. C. baccatum genotypes were the most resistant with no infection.

Aggressiveness of Colletotrichum capsici isolates. Differences among pathogen isolates in quantitative level of infection occurred in all genotypes of Capsicum spp. except for Capsicum baccatum, where no

Table 2. Mean percent lesion size of 9 chili genotypes at 9 days after inoculation with 11 Colletotrichum capsici isolates $^{\mathrm{x}}$

\begin{tabular}{|c|c|c|c|c|c|c|c|c|c|c|}
\hline \multirow[b]{2}{*}{ Isolates } & \multicolumn{3}{|c|}{ Capsicum annuum } & \multicolumn{3}{|c|}{ C. baccatum } & \multicolumn{2}{|c|}{ C. chinense } & \multirow{2}{*}{$\frac{\text { C. frutescens }}{\text { Kheenoo Suan }}$} & \multirow[b]{2}{*}{ Meany } \\
\hline & Bangchang & $83-168$ & Jinda & PBC 80 & PBC 81 & PBC 1422 & PBC932 & C04714 & & \\
\hline $\mathrm{F} 4-2 \mathrm{C}$ & $11 \mathrm{st}$ & $100 \mathrm{a}$ & 27 klmno & 0 & 0 & 0 & $0 \mathrm{u}$ & $11 \mathrm{t}$ & $100 \mathrm{a}$ & $44 \mathrm{~F}$ \\
\hline F4-5A & $32 \mathrm{jklm}$ & $100 \mathrm{a}$ & 35 hijkl & 0 & 0 & 0 & $0 \mathrm{u}$ & $30 \mathrm{klm}$ & $100 \mathrm{a}$ & $54 \mathrm{D}$ \\
\hline F7-3A & 36 hijk & $62 \mathrm{def}$ & 19 opqr & 0 & 0 & 0 & $0 \mathrm{u}$ & $0 \mathrm{u}$ & $95 \mathrm{~b}$ & $28 \mathrm{G}$ \\
\hline F8-1A & $66 \mathrm{~d}$ & $100 \mathrm{a}$ & 27 klmno & 0 & 0 & 0 & $53 \mathrm{gf}$ & $55 \mathrm{f}$ & $100 \mathrm{a}$ & $75 \mathrm{~A}$ \\
\hline F8-2A & 29 klmn & $100 \mathrm{a}$ & 25 lmnop & 0 & 0 & 0 & $22 \mathrm{mnopq}$ & 23 mnop & $81 \mathrm{~b}$ & $51 \mathrm{DE}$ \\
\hline F8-3A & 27 klmno & $100 \mathrm{a}$ & 25 lmnop & 0 & 0 & 0 & $30 \mathrm{klm}$ & 21 nopq & $100 \mathrm{a}$ & $58 \mathrm{C}$ \\
\hline F8-3B & 56 ef & $100 \mathrm{a}$ & 35 hijkl & 0 & 0 & 0 & 18 pqrs & 27 klmnop & $100 \mathrm{a}$ & $65 \mathrm{~B}$ \\
\hline $\mathrm{F} 8-4 \mathrm{C}$ & 19 opqr & $100 \mathrm{a}$ & $41 \mathrm{hij}$ & 0 & 0 & 0 & 59 def & 25 lmnop & $100 \mathrm{a}$ & $66 \mathrm{~B}$ \\
\hline F8-5A & $68 \mathrm{~d}$ & $100 \mathrm{a}$ & 29 klmn & 0 & 0 & 0 & $0 \mathrm{u}$ & $34 \mathrm{ijkl}$ & $100 \mathrm{a}$ & $60 \mathrm{C}$ \\
\hline F8-5B & 35 hijkl & 42 hi & 25 lmnop & 0 & 0 & 0 & 33 ijkl & 27 klmnop & $100 \mathrm{a}$ & $48 \mathrm{E}$ \\
\hline SKP16 & $12 \mathrm{rst}$ & $65 \mathrm{de}$ & 15 qrst & 0 & 0 & 0 & $0 \mathrm{u}$ & $0 \mathrm{u}$ & $45 \mathrm{gh}$ & $15 \mathrm{H}$ \\
\hline $\operatorname{Mean}^{\mathrm{z}}$ & $35 \mathrm{C}$ & $89 \mathrm{~B}$ & $27 \mathrm{D}$ & 0 & 0 & 0 & $12 \mathrm{~F}$ & $19 \mathrm{E}$ & $98 \mathrm{~A}$ & $\ldots$ \\
\hline
\end{tabular}

x Values for pathogen isolate-host genotype combinations with the same letter are not significantly different $(P=0.05)$ based on Duncan's multiple range test.

${ }^{y}$ Means for isolates exclude reactions of $C$. baccatum genotypes. Values in the column with the same letter are not significantly different $(P=0.05)$ based on

Duncan's multiple range test.

${ }^{z}$ Values in the row with the same letter are not significantly different $(P=0.05)$ based on Duncan's multiple range test.

Table 3. Anthracnose severity scores on chili fruits, chili resistance levels, and symptom description

\begin{tabular}{lll}
\hline Score & \multicolumn{1}{c}{ Resistance level } & \\
\hline 0 & HR, highly resistant & No infection \\
1 & R, resistant & $1-2 \%$ of the fruit area shows necrotic lesion or a larger water-soaked lesion surrounding the infection site \\
3 & MR, moderately resistant & $>2-5 \%$ of the fruit area shows necrotic lesion, acervuli may be present, or water-soaked lesion up to $5 \%$ of the fruit \\
& & surface
\end{tabular}


infection occurred (Table 2). There were significant differences in the level of aggressiveness of the isolates, with F8-1A being the most aggressive, resulting in $75 \%$ mean lesion size on all host genotypes (excluding the $C$. baccatum genotypes), followed by F8-4C (66\%) and F8$3 \mathrm{~B}(65 \%)$. The least aggressive isolates were SKP16 (15\%) and F7-3A (28\%), which did not infect any of the $C$. chinense and $C$. baccatum genotypes.

Disease scales for Colletotrichum capsici infection of Capsicum spp. A set of disease ratings of 0 to 9 was established based on the percent lesion size relative to the overall size of the fruit, appearance of infected tissue (necrotic or water soaked), and the presence of acervuli. A rating of 0 was no infection and 9 was infection greater than $25 \%$ (Table 3 ). A set of disease scales with disease severity based on the 0-to-9 scale was developed for fruit types of Capsicum annuum, C. baccatum, C. frutescens, and C. chinense (Fig. 1). These scales compensated for the different size of fruit and size of lesion. C. chinense genotypes consisted of small circular fruit while $C$. baccatum genotypes were large, elongated, and wide fruit.

Using the scales, $C$. frutescens and $C$. annuum genotypes were found to be highly susceptible, a rating of 9 , to most or all pathogen isolates (Table 4). Both $C$. chinense genotypes $\mathrm{C} 04714$ and $\mathrm{PBC} 932$ reacted with ratings from 0 to 9 depending on the pathogen isolate. C. baccatum genotypes were the most resistant, with no infection (rating 0 ) by any isolate.

Pathotype identification of Colletotrichum capsici. Based on the qualitative differential reactions (Table 4), three pathotypes of Colletotrichum capsici (PCc) were identified, PCc1, PCc2, and $\mathrm{PCc} 3$. The $\mathrm{PCc} 1$ group contained isolates F8-1A, F8-2A, F8-3A, F8-3B, F8-4C, and F8-5B. These were able to infect all genotypes of Capsicum annuum, C. frutescens, and $C$. chinense, with disease scores ranging from 5 to 9, but did not infect $C$. baccatum. The $\mathrm{PCc} 2$ group of isolates $(\mathrm{F} 4-2 \mathrm{C}$, F4-5A, and F8-5A) was able to infect all genotypes of $C$. annuum, C. frutescens, and $C$. chinense $\mathrm{C} 04714$ but not $C$. chinense PBC932 or any genotype of $C$. baccatum. The PCc3 isolates (F7-3A and SKP16) infected only genotypes of $C$. annuum and $C$. frutescens but did not infect genotypes of $C$. baccatum and $C$. chinense.

\section{DISCUSSION}

Three pathotypes (PCc1, PCc2, and PCc3) of Colletotrichum capsici were identified from 11 isolates collected from the western and northern districts of Thailand, based on qualitative differences in infection on Capsicum chinense genotypes PBC932 and C04714. Sharma et al. (8) reported the existence of 15 pathotypes of Colletotrichum capsici from 35 isolates collected in the Himachal Pradesh area of northern India. In contrast, these isolates were screened on nine genotypes of Capsicum annuum where "pathotype" differences were based on quantitative differences in the level of infection between cultivars. However, according to Taylor and Ford (9), a pathotype is defined as a group of isolates distinguished from others of the same species by a qualitative difference in disease severity. A quantitative difference in infection reflects the natural distribution of aggressiveness within a population, ranging from low to high. Therefore, it is most likely that Sharma et al. (8) only observed differences in aggressiveness between isolates and did not identify true pathotype differences of Colletotrichum capsici. Moreover, only Capsicum annuum genotypes were used to screen for differences in infection. C. annuum genotypes are known to be very susceptible to anthracnose (11). The C. annuum genotype Bangchang used in the current study

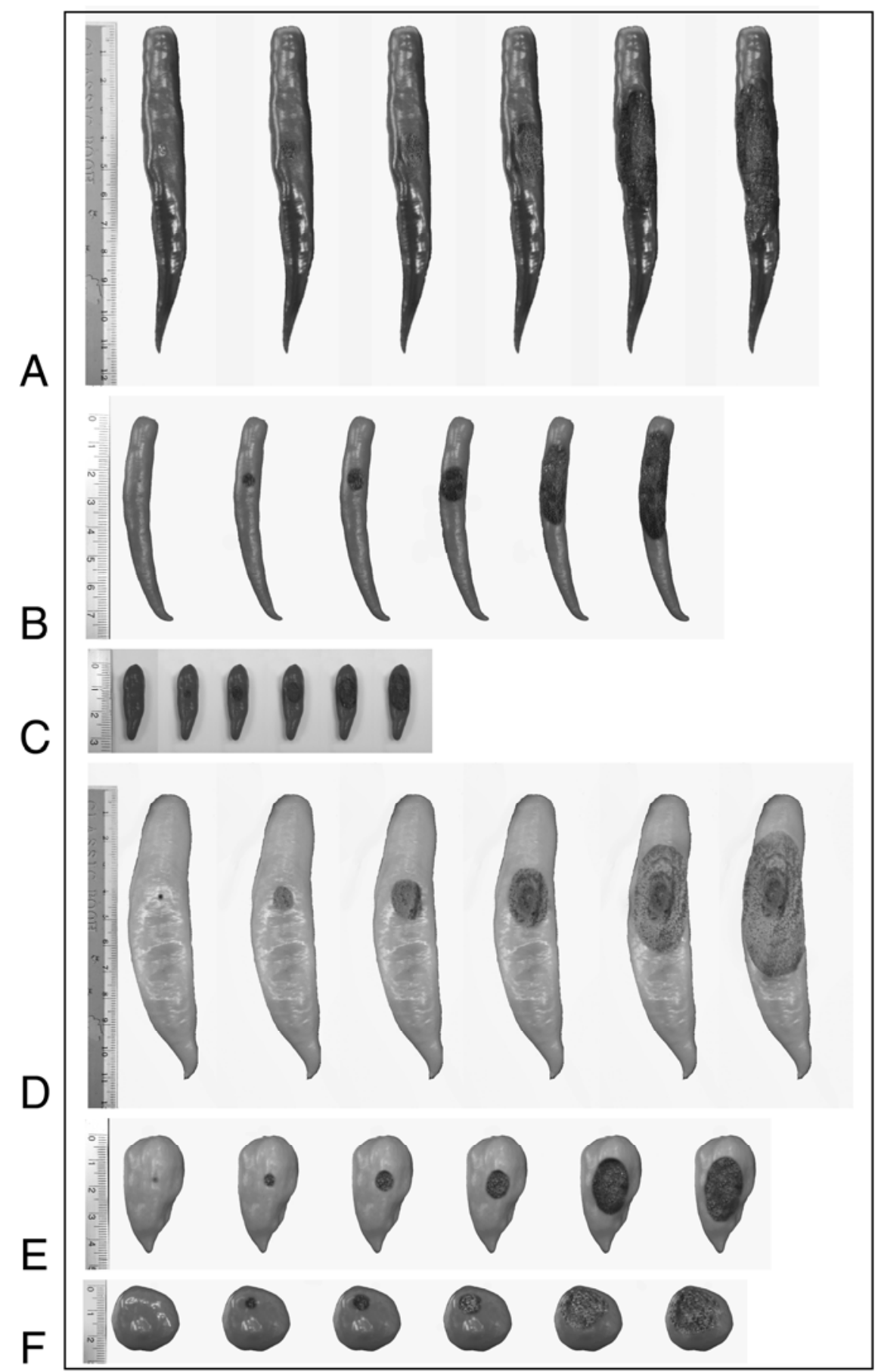

Fig. 1. Disease diagrams representing degrees of infection ranging from scores of 0 to 9 (from left to right in each row) in various chili fruit types: A, large and elongate type as Capsicum annuum Bangchang; B, large Kheenoo type as $C$. аnnuum Jinda and 83-168; C, small Kheenoo type as $C$. frutescens Kheenoo Suan; D, C. baccatum type; E, conical type as $C$. chinense $\mathbf{C} 04714$; and $\mathbf{F}$, round type as $C$. chinense PBC932. 
Table 4. Anthracnose severity evaluated as disease scores on a 0 -to-9 scale and pathotypes of the 11 Colletotrichum capsici isolates $^{\mathrm{z}}$

\begin{tabular}{|c|c|c|c|c|c|c|c|c|c|c|}
\hline \multirow[b]{2}{*}{ Isolates } & \multicolumn{3}{|c|}{ Capsicum annuum } & \multicolumn{3}{|c|}{ C. baccatum } & \multicolumn{2}{|c|}{ C. chinense } & \multirow{2}{*}{$\frac{C . \text { frutescens }}{\text { Kheenoo Suan }}$} & \multirow[b]{2}{*}{ Pathotype } \\
\hline & Bangchang & 83-168 & Jinda & PBC80 & PC81 & PBC1422 & PBC932 & C04714 & & \\
\hline F8-1A & 9 & 9 & 9 & 0 & 0 & 0 & 9 & 9 & 9 & PCc 1 \\
\hline F8-2A & 9 & 9 & 9 & 0 & 0 & 0 & 7 & 7 & 9 & PCc 1 \\
\hline F8-3A & 9 & 9 & 9 & 0 & 0 & 0 & 9 & 7 & 9 & PCc 1 \\
\hline F8-3B & 9 & 9 & 9 & 0 & 0 & 0 & 7 & 9 & 9 & PCc 1 \\
\hline F8-4C & 7 & 9 & 9 & 0 & 0 & 0 & 9 & 9 & 9 & PCc 1 \\
\hline F8-5B & 9 & 9 & 9 & 0 & 0 & 0 & 9 & 9 & 9 & PCc 1 \\
\hline F4-2C & 5 & 9 & 9 & 0 & 0 & 0 & 0 & 5 & 9 & PCc 2 \\
\hline F4-5A & 9 & 9 & 9 & 0 & 0 & 0 & 0 & 9 & 9 & $\mathrm{PCc} 2$ \\
\hline F8-5A & 9 & 9 & 9 & 0 & 0 & 0 & 0 & 9 & 9 & PCc 2 \\
\hline F7-3A & 9 & 9 & 9 & 0 & 0 & 0 & 0 & 0 & 9 & PCc 3 \\
\hline SKP16 & 5 & 9 & 5 & 0 & 0 & 0 & 0 & 0 & 9 & PCc 3 \\
\hline
\end{tabular}

${ }^{\mathrm{z}}$ Disease scores are as described in Table 3.

showed susceptible scores from 5 to 9 depending on the aggressiveness of the isolate.

Infection of fruit that results in blemishes and lesions diminishes the marketability of the fruit. If more than 5\% infection of the fruit surface was infected, then this was rated as a susceptible reaction (disease score of 5 to 9). Infection levels greater than $25 \%$ were assigned the highest rating because such levels lead to severe impact on the survivability of the fruit, with high levels of inoculum being produced from acervuli. Although resistant (score 1) and moderately resistant (score 3) host fruit reactions were not observed for Colletotrichum capsici infection, these host reactions have been observed with low-aggressive isolates of C. capsici in Australia and were more common in genotypes infected with $C$. acutatum (unpublished data). In some genotypes, particularly of Capsicum baccatum infected by Colletotrichum acutatum (unpublished data), a resistant reaction (scores 1 and 3) on the fruit was observed as water-soaked lesions surrounding the infection site, with a notable absence of acervuli.

Knowledge of both existence of pathotypes and level of aggressiveness of the pathogen isolates is extremely important when choosing appropriate isolates to screen germplasm and lines for resistance in plant breeding programs. Further work should include screening of more isolates, particularly from other chili-growing regions of Thailand, on the differential genotypes Capsicum chinense PBC932 and C04714.

\section{ACKNOWLEDGMENTS}

We thank the National Center for Genetic Engineering and Biotechnology, National Science and Technology Development Agency for financial support for a Master's student scholarship and other project support. Field experiments were supported by the Tropical Vegetable Research Center, Kasetsart University, Kamphaeng Saen Campus.

\section{LITERATURE CITED}

1. FAOSTAT. 2008. Production data. Available online at: http://faostat.fao.org/

2. Kanchana-udomkan, C., Taylor, P. W. J., and Mongkolporn, O. 2004. Development of a bioassay to study anthracnose infection of chili fruit caused by Colletotrichum capsici. Thai J. Agric. Sci. 37:293-297.

3. Kirkpatrick, L. A., and Feeney, B. C. 2006. A Simple Guide to SPSS for Windows for Version 12.0 \& 13.0. Thomas Wadsworth, Belmont, NY.

4. Mustafa, U., Ali, M., Satapornvorasak, T., and Dissataporn, O. 2006. Thailand. Pages 197245 in: Chili (Capsicum spp.) Food Chain Analysis: Setting Research Priorities in Asia. M. Ali, ed. World Veg. Cent. Tech. Bull. No. 38
5. Pakdeevaraporn, P., Wasee, S., Taylor, P. W. J., and Mongkolporn, O. 2005. Inheritance of resistance to anthracnose caused by Colletotrichum capsici in Capsicum. Plant Breed. 124:206-208.

6. Photita, W., Lumyong, S., Lumyong, P., McKenzie, E. H. C., Ford, R., Taylor, P. W. J., and Hyde, K. D. 2005. Morphological and molecular characterisation of Colletotrichum species from herbaceous plants in Thailand. Fungal Divers. 18:117-133.

7. Poonpolgul, S., and Kumphai, S. 2007. Chili pepper anthracnose in Thailand. Page 23 in: First Int. Symp. Chili Anthracnose. Convention Center, Seoul National University, Korea.

8. Sharma, P. N., Kaur, M., Sharma, O. P., Sharma, P., and Pathania, A. 2005. Morphological, pathological and molecular variability in Colletotrichum capsici, the cause of fruit rot of chilies in the subtropical region of north-western India. J. Phytopathol. 153:232237.

9. Taylor, P. W. J., and Ford, R. 2007. Diagnostics, genetic diversity and pathogenic variation of Ascochyta blight of cool season food and feed legumes. Eur. J. Plant Pathol. 119:127133.

10. Than, P. P., Jeewon, R., Hyde, K. D., Pongsupasamit, S., Mongkolporn, O., and Taylor, P. W. J. 2008. Characterization and pathogenicity of Colletotrichum species associated with anthracnose on chili (Capsicum spp.) in Thailand. Plant Pathol. 57:562-572.

11. Yoon, J. B., Yang, D. C., Lee, W. P., Ahn, S. Y and Park, H. G. 2004. Genetic resources resistant to anthracnose in the genus Capsicum. J. Korean Soc. Hortic. Sci. 45:318-323. 